\title{
Respiratory impairment in Niemann-Pick B disease: two case reports and review for the pulmonologist
}

\author{
Thibaut Capron ${ }^{1}$, Youssef Trigui ${ }^{2}$, Clarisse Gautier ${ }^{1}$, Basile Puech ${ }^{3}$, Pascal Chanez ${ }^{2}$ \\ and Martine Reynaud-Gaubert ${ }^{1}$ \\ ${ }^{1}$ Centre de compétences national maladies pulmonaires rares - équipe de \\ transplantation pulmonaire - Assistance Publique - Hôpitaux de Marseille ; CHU \\ Nord ; Aix-Marseille Université, Marseille, France \\ ${ }^{2}$ Clinique des bronches, allergies et sommeil - Assistance Publique - Hôpitaux de \\ Marseille ; CHU Nord ; Aix-Marseille Université, Marseille, France \\ ${ }^{3}$ Service de radiologie - Assistance Publique - Hôpitaux de Marseille ; CHU Nord ; \\ Aix-Marseille Université, Marseille, France
}

\section{Abstract: 169 \\ Word Count: 2641}

Keywords: Niemann-Pick, Acid sphingomyelinase deficiency, pulmonary storage disorder, lung transplant, hemoptysis.

\section{Corresponding author}

Thibaut Capron

Centre de compétences national maladies pulmonaires rares équipe de transplantation pulmonaire Assistance Publique des Hôpitaux de Marseille - CHU Nord Chemin des Bourrely 13915 Marseille Cedex 20

\section{FRANCE}

E-mail: thibaut.capron@ap-hm.fr, thibaut.capron@ gmail.com

Phone: +33 (0) 6255500 23, Fax: +33 (0) 491966140 


\begin{abstract}
Acid sphingomyelinase deficiency (ASMD), also called Niemann-Pick disease, is a storage disorder with pulmonary involvement but few respiratory symptoms in adults. However, the disease may evolve towards clinically relevant respiratory symptoms with referral to the pulmonologist for management and care. Based on two case reports illustrating respiratory impairment, the aim of this work was to review clinical features, diagnosis, respiratory prognostic and therapeutics for the pulmonologist.

Overall, storage disorder should be suspected in the presence of hepatosplenomegaly and interstitial lung disease. Concomitant thrombopenia or hyperlipidemia should also draw attention.

Following recent consensus guidelines, diagnosis is based on enzyme assay for ASM activity in blood, with subsequent gene sequencing once the biochemical diagnosis has been confirmed. Disease is slowly progressive and the main causes of death are respiratory and liver failure. Presence of emphysema lesions or worsening of respiratory symptoms should call for the intensification of treatment. Though enzyme replacement therapy is a promising way of development, lung transplantation might be considered for these patients in the absence of contraindication.
\end{abstract}




\section{Introduction}

Acid sphingomyelinase deficiency (ASMD) is a rare lysosomal storage disorder caused by autosomal recessive genetic mutations in the SMPD1 gene. It has been historically termed as Niemann-Pick disease. Patients reaching adulthood often exhibit interstitial lung disease although rarely presenting respiratory symptoms. This is a reason why this disease remains poorly known from pulmonologists, whereas new diagnostic recommendations and promising therapeutics are rising. We present two case reports illustrating respiratory impairment in such patients with referral to the pulmonologist. We briefly review and discuss typical aspects of the disease and related up-to-date challenges for the pulmonologist.

\section{Case report \#1:}

A 50-year-old man was referred to our center for the worsening of his respiratory status and assessment for lung transplantation.

He had been diagnosed with Niemann-Pick disease type B six years ago, following the exploration of increased dyspnea revealing an interstitial lung disease. He reported only a 20 pack-years smoking history as background. The computed tomography (CT) scan found an interstitial pattern and revealed hepatosplenomegaly for which he was referred to the hematologist. Vacuolated macrophages were found in bone marrow and a storage disorder was suspected. In order to ascertain diagnosis, the patient benefited from wedge lung resection, which confirmed interstitial infiltration with lipid-laden cells compatible with Gaucher's disease. Deficiency of the acid sphyngomyelinase enzyme activity in blood was observed and the final diagnosis of Niemann-Pick type B disease was set. Genetic analysis of the SMPD1 gene yielded the homozygous deletion p.R610, without family background for such a disease. After the diagnosis, the patient stopped follow-up for 5 years. He was referred back to pulmonologist due to progressively increased dyspnea. He quit smoking one year before, required continuous $2 \mathrm{~L} / \mathrm{min}$ oxygen support and was treated with $10 \mathrm{mg}$ corticosteroid per day. According to this worsening respiratory condition, he was referred to our lung transplantation center.

On admission, blood arterial gases revealed hypoxemia $\left(\mathrm{PaO}_{2} 55 \mathrm{mmHg}\right)$ without hypercapnia. Pulmonary function testing evidenced a moderate restriction (Forced Vital Capacity FVC $75 \%$ theorics) with lowered diffusion capacity for carbon monoxide (DLCO $45 \%$ theorics). 6-minutes walk distance test (6MWD) performed under continuous $4 \mathrm{~L} / \mathrm{min}$ oxygen support showed decreased saturation down to $84 \%$ with $480 \mathrm{~m}$ distance walked. Thoracic CT scan 
found diffuse abnormalities along a cranio-caudal gradient. They consisted of ground-glass opacities with intermixed intralobular lines and interlobular septal thickening (Figure 1a-c), characteristic of the so-called "crazy-paving" pattern. Infra-centimetric mediastinal nodes were also noted. Global comparison with anterior results drew the conclusion of a smooth slow deterioration.

On the cardiovascular side, echocardiography did not show any sign of cardiac dysfunction or pulmonary hypertension. Coronarography was normal. Arterio-venous vascular axes were free from significant lesion. Liver function was normal and portal hypertension was ruled out. Biology found isolated low platelet count to $93 \mathrm{G} / \mathrm{L}$ and hyperlipidemia with high level of triglycerides and low level of high-density lipoprotein (HDL). Renal and hepatic functions were normal. Previous abdominal CT scan found spleen nodules with, on ultrasound, an aspect of angioma.

Finally the evaluation did find indication for lung transplantation with a very motivated patient reporting major alteration of his quality of life. No contraindications were noted. Information and psychological support were provided to the patient and he has been placed on the waiting list for double lung transplantation 3 months later.

\section{Case report \#2 :}

A 44-years old caucasian man referred to pulmonologist with moderate hemoptysis. He had never smoked and had been diagnosed with Niemann-Pick disease type B at the age of 3 years old, following the exploration of hepatosplenomegaly. The patient had only minor dyspnea for important efforts as respiratory symptoms, and lung interstitial pattern was discovered on CT 5 years before. Genetic analysis of SMPD1 is in progress and there was no family history of the disease. The patient also had symptomatic hiatal hernia treated by proton pump inhibitors.

He consulted for an intermittent hemoptoic productive cough since nearly one month, without thoracic pain nor fever. He was not febrile, and clinical examination did not find signs of respiratory failure. Blood pressure and oxygen saturation were normal and cardio-pulmonary auscultation did not reveal any significant findings.

Laboratory blood examinations found preserved hemoglobin and low platelet count to $111 \mathrm{G} / \mathrm{L}$, reticulocyte count was slightly elevated to $91 \mathrm{G} / \mathrm{L}$. C reactive protein, renal function and coagulation were normal. Dyslipidemia was noted with slightly elevated triglycerides and low HDL cholesterol level. 
Thoracic CT scan showed bilateral lower lobes ground glass opacities associated with intermixed intralobular and interlobular lines in upper lobes (Figure 2). Emphysema lesions were observed in apical regions whereas very distal bronchiolectasis were noticeable in basal lungs (Figure 3).

Because of profuse basal ground glass opacities in relation with the chronic disease, no additional sign of alveolar hemorrhage could be diagnosed on CT. There was neither vascular target for embolization nor evidence for the origin of bleeding.

Flexible bronchoscopy with bronchoalveolar lavage (BAL) revealed an active bleeding from the right lower lobe (segmental lateral basal B9). A radial endobronchial ultrasound probe was used to confirm the absence of endobronchial lesions in this segment and in the upstream segment. BAL was macroscopically hemorrhagic and showed elevated cell count. The BAL cultures were negative for bacteria, mycobacteria and fungi. No malignant cells were found. Differential cell count provided $8 \%$ neutrophils, $3 \%$ lymphocytes and $89 \%$ macrophages comprising $7 \%$ hemosiderin-laden macrophages with a Golde score of 95 .

The diagnosis of alveolar hemorrhage was retained and the patient started therapy with oral prednisolone $1 \mathrm{mg} / \mathrm{kg}$ per day without any improvement.

Pulmonary function tests were unchanged as compared to 2014 with preserved volumes (FVC $93 \%$ theorics) and mild alteration of diffusing capacity (DLCO $54 \%$ theorics). The patient did not present any desaturation in 6MWD.

Immunological blood analyses including anticytoplasmic antibodies (myeloperoxidase and proteinase 3), antiglomerular basal membrane antibodies, antinuclear antibodies, rheumatoid factor and antibodies to citrullinated peptide were negative. No drugs could be incriminated in the alveolar hemorrhage. Specifically, there was no current epistaxis or gingival hemorrhage episodes that could point out a role of the relative thrombocytopenia.

Finally, the patient had isolated recurrent low-abundance hemoptysis with signs of idiopathic alveolar hemorrhage and impairment of his quality of life. In the absence of specific treatment, close follow-up was proposed to the patient. 


\section{Review of ASMD and discussion}

ASMD is a lysosomal storage disorder commonly known as Niemann-Pick disease type A (NPD A) and Niemann-Pick disease type B (NPD B) (1). NPD A patients have mainly neurological impairment and a poor prognosis with a median lifespan of 3 years after birth. In contrast, NPD B patients reach adulthood, show very variable disease course and a broader spectrum of clinical manifestations including pulmonary involvement $(2,3)$. While typical NPD B patients do not exhibit neurological manifestations, a subset of patients termed "variant NPD B" may have mild-to-severe peripheral neurological symptoms (4). The Niemann-Pick disease type $\mathrm{C}$, initially grouped with ASMD, is however driven by other pathogenesis, different genetic disorders and central nervous system is mainly involved. NPD A and B classifications were historically based on clinical presentation but owing to the various manifestations and severity levels of the disease, it might be more appropriated to report them as pathogenenesis-based ASMD disease (4). The estimated incidence of ASMD is approximately 0.5 per 100000 births (5).

Pathogenesis is caused by mutations in the SMPD1 gene, coding for acid sphingomyelinase. Such mutation results in lower enzymatic activity and abnormal accumulation of lipids in cells, predominantly macrophages and hepatocytes. This deposition disease evolves slowly and progressively and may impair multiple organs. Histopathological analyses typically show lipid-laden cells, so-called "foam cells" in liver, spleen, lymph nodes, adrenal cortex, lungs and/or bone marrow. Specifically for the lung, lipid-laden cells accumulate in the alveolar septa, bronchial walls and subpleural spaces (6).

A major challenge for the pulmonologist is to identify ASMD patients (7), due to the scarcity and the heterogeneity of the disease. Overall, storage disorder should be suspected in patients presenting with hepatosplenomegaly and interstitial lung disease (Figure 1). Indeed, such patients almost systematically have pulmonary involvement detectable by imaging, but without respiratory symptoms. In the largest prospective case series, common symptoms were splenomegaly (78 \%) and hepatomegaly (73\%). The most common complaints were bleeding (49\%, recurrent epistaxis in $29 \%$ ), pulmonary infections (42\%), shortness of breath (42\%) and joint/limb pain (39\%) (8). Clinical manifestations may also include developmental delay or retina cherry-red maculae (9). Thrombocytopenia and mixed dyslipidemia are typical biological features reported in NPD B patients $(8,10)$. This dyslipidemia is characterized by low 
high-density-lipoprotein (HDL) cholesterol and high levels of low-density-lipoprotein (LDL) cholesterol and triglycerides.

Respiratory impairment ranges from absence of symptoms to rare chronic failure $(11,12)$. Mainly reported respiratory symptoms are dyspnea on mild exertion and recurrent infections. As illustrated in case \#1, the disease may evolve towards oxygen requirement and reduced tolerance to exercise. Some studies reported that pulmonary disease is the most debilitating clinical feature in NPD B patients (10) and retrospective compelling of the data from ASMD deceased patients found that overall leading causes of death were respiratory failure and liver failure (27\% each) (13).

Interstitial lung disease is the most prominent feature found in prospective surveys, concerning $90-98 \%$ of NPD B patients $(8,14)$, predominantly in the lower lobes. However the severity of imaging assessment does not correlate with the lack of alteration of pulmonary function tests (14). Indeed, pulmonary function tests can demonstrate normal lung volumes or mild restrictive pattern, with a slight alteration of the diffusion capacity for carbon monoxide. Longitudinal follow-up highlighted a gradual but slow deterioration of pulmonary function over time, reflecting a slow progression of the disease $(2,10)$.

Bronchoalveolar lavage is helpful to highlight lipid-laden macrophages characteristic of lipoid pneumonia (15) but is not specific for Niemann-Pick disease. Lung biopsy samples allow for studying morphology and topography of the infiltration with lipoid cells, which is necessary for the pathologist to argue for diagnosis of metabolic disorder (16). Typical features are large multivacuolated histiocytes staining deep blue with MGG, so-called Niemann-Pick cells or "sea-blue histiocytes".

Chest CT identifies ground-glass opacities, mild smooth interlobular septal thickening and intralobular lines, predominantly in the lower lung regions. Such mixed observations form the so-called "crazy-paving" pattern $(14,17)$.

For the first patient presented above, CT imaging during an acute dyspnea episode one year before is shown on figure 1-d. Numerous bilateral ground-glass opacities were visible and had disappeared one year after (figure 1-b). Clinical evolution was spontaneously favorable without any treatment in the absence of positive microbiological documentation. This suggests exacerbations of the disease with radiological reversible lesions, which are not described in 
ASMD and may be interpreted as low respiratory tract infection episode in view of the symptoms and the chest radiograph.

The second patient had unusual lesions on CT imaging: apical emphysema and very distal bronchiolectasis in lower lobes (Figure 3). Comparison with previous CT performed 5 years before demonstrated slow progression of these anomalies. Bronchial distortion and subpleural emphysema-like lesions were also identifiable in our first patient (Figure 1-a). Following the model of macrophage-related interstitial lung diseases in cigarette smokers, macrophage dysfunction can induce emphysema or fibrosis (6). Our cases may suggest an additional alveolar macrophage dysfunction, which could be related to the progression of respiratory impairment.

Main features of the two cases presented above are summarized in table 1. These reports highlight the concerns for the pulmonologist in ASMD disease, especially when respiratory impairment does develop. Actually, several series of patients identified respiratory impairment as a major cause of death $(13,18)$. Onset of respiratory symptoms may thus be viewed in favor of the intensification of the treatment and care.

The clinical presentation depicted earlier is highly suggestive of lysosomal storage disorder but is shared by Gaucher's disease. Hepatosplenomegaly and pulmonary impairment may also occur, with abnormal pulmonary function tests and similar interstitial pattern on CT (19). As illustrated in our first case report, histopathological analyses may also fail to discriminate the two diseases. Following recent consensus guidelines, first-intention measurement of acid sphingomyelinase enzymatic activity provides the differential diagnosis (20). This can be reliably done in peripheral blood lymphocytes or cultured skin fibroblasts. Simultaneous determination of the glucocerebrosidase activity in blood, which is specifically lowered in Gaucher's disease, is also recommended. Genetic detection of pathogenic SMPD1 variants may bring diagnostic information but should be reserved to second-intention analysis after enzymatic diagnosis, due to the variety of mutations of unknown significance. These diagnostic recommendations are summarized as an algorithm in recent consensus expert guidelines (20).

Management of ASMD is currently based on symptomatic treatment and supportive care. Whole lung lavage, based on pulmonary alveolar proteinosis protocols, has shown efficacy in few cases reports but remains a high-risk procedure with only temporary benefit $(21,22)$. Importantly, splenectomy has to be avoided as it may worsen hyperlipidemia profile (8), 
whereas updated vaccination is warranted. Potential effect of steroids remains an open question in guidelines for clinical management (23).

Promising new enzyme replacement therapies are rising with the potential to modify the natural history of the disease. Results of a phase 1 trial of recombinant human acid shingomyelinase in ASMD patients have been published in 2016 (24) and results of phase 1b study are about to be announced (25). Clinical development is continuing with an ongoing phase $2 / 3$ trial in ASMD adults (26).

It is worthwhile to notice that our first patient was contraindicated to enzyme replacement therapy trial because of the severity of his respiratory condition, raising again interest in an earlier diagnosis.

Another end-stage treatment that could be considered by pulmonologist is lung transplantation, as illustrated in case \#1. Very recently, a first case reporting such procedure in a patient with NPD B was published. Diffuse alveolar damage occurred on the lung allograft, attributed to major influx of recipient-derived lipid-laden macrophages and patient died at day 29 (27). While it was proposed that such reaction could be prevented using enzyme replacement therapy (25), a second case of successful lung transplantation was reported (28). The authors applied standard immunosuppression protocol and emphasized that normal liver function plays a fundamental role in protecting the lung allograft. Lung transplantation candidates should thus be carefully checked-up for liver function and portal hypertension.

Interestingly, these two patients showing severe NPD B also presented upper-lobe emphysema, consistent with our two cases descriptions of severity. Moreover, these recent publications on lung transplantation in NPD B confirm the growing referral of these patients to the pulmonologist.

\section{Conclusion}

Acid sphingomyelinase deficiency is a rare disease with growing concerns for the pulmonologist. Almost all patients present asymptomatic interstitial lung disease and few of them exhibit respiratory symptoms for which they can be referred to the pulmonologist. Early diagnosis is still currently limited by the lack of awareness of healthcare professionals. Storage disorder should be suspected when combined with hepatosplenomegaly and typical biologic abnormalities; diagnosis should be confirmed following recent expert consensus guidelines based on first-intention measurement of enzymatic activity.

The disease is slowly but irremediably progressive and this has to be taken into account in order to adapt therapeutics or refer the patients for lung transplantation. Unlike in other interstitial 
pulmonary diseases such as fibrosis, no clear steeper degradation signal is currently appreciable. However, cases discussed in this review suggest that emphysema or marked bronchiolectasis may be a marker for more severe respiratory impairment.

Enzyme replacement therapy is very promising and involves pulmonologist for early diagnosis, follow-up of respiratory function and referral to expert centers. Potentially, efficient therapy improvements would intensify discussions around newborn screening for such storage disorders in the future (29).

\section{Acknowledgments}

We acknowledge fruitful discussions on chest imaging with Pr J.-Y. Gaubert and Dr P. Habert, but also concerning clinical management of these cases with Dr K. Mazodier, Dr C. Stavris, Dr G. Thomas, Dr J.-B. Rey, Dr E. Prud'homme and J. Bermudez. 


\section{Figures}

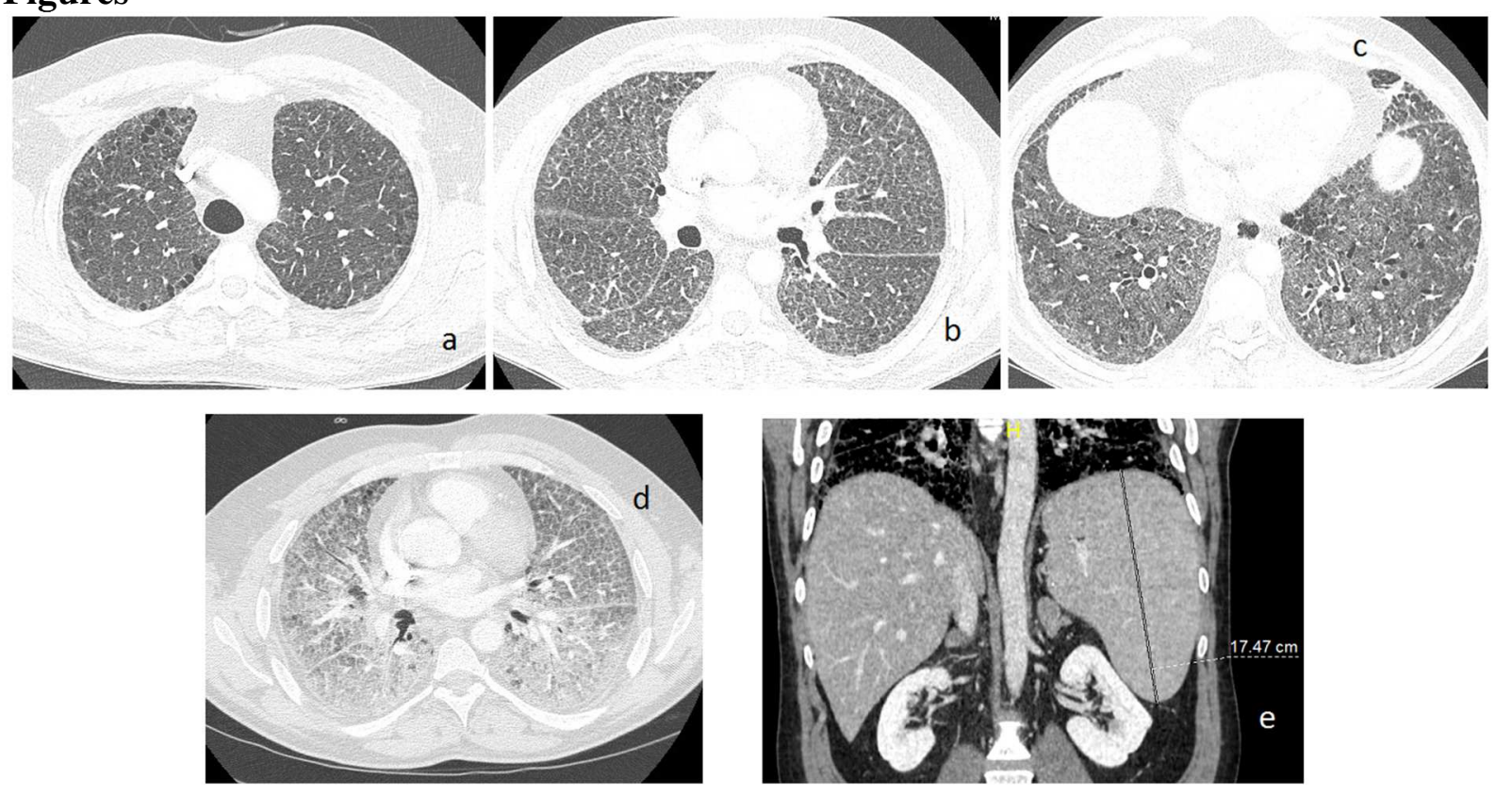

Figure 1: computed tomography of case\#1. Top: current chest slices at (a) upper lobes showing subpleural emphysema-like lesions, (b) middle-lung showing diffuse mixed interlobular and intralobular lines,(c) lower lobes with bilateral ground glass opacities.

Bottom: (d) chest image during exacerbation one year before with markedly profuse ground glass opacities. (e) abdominal slice showing splenomegaly. 

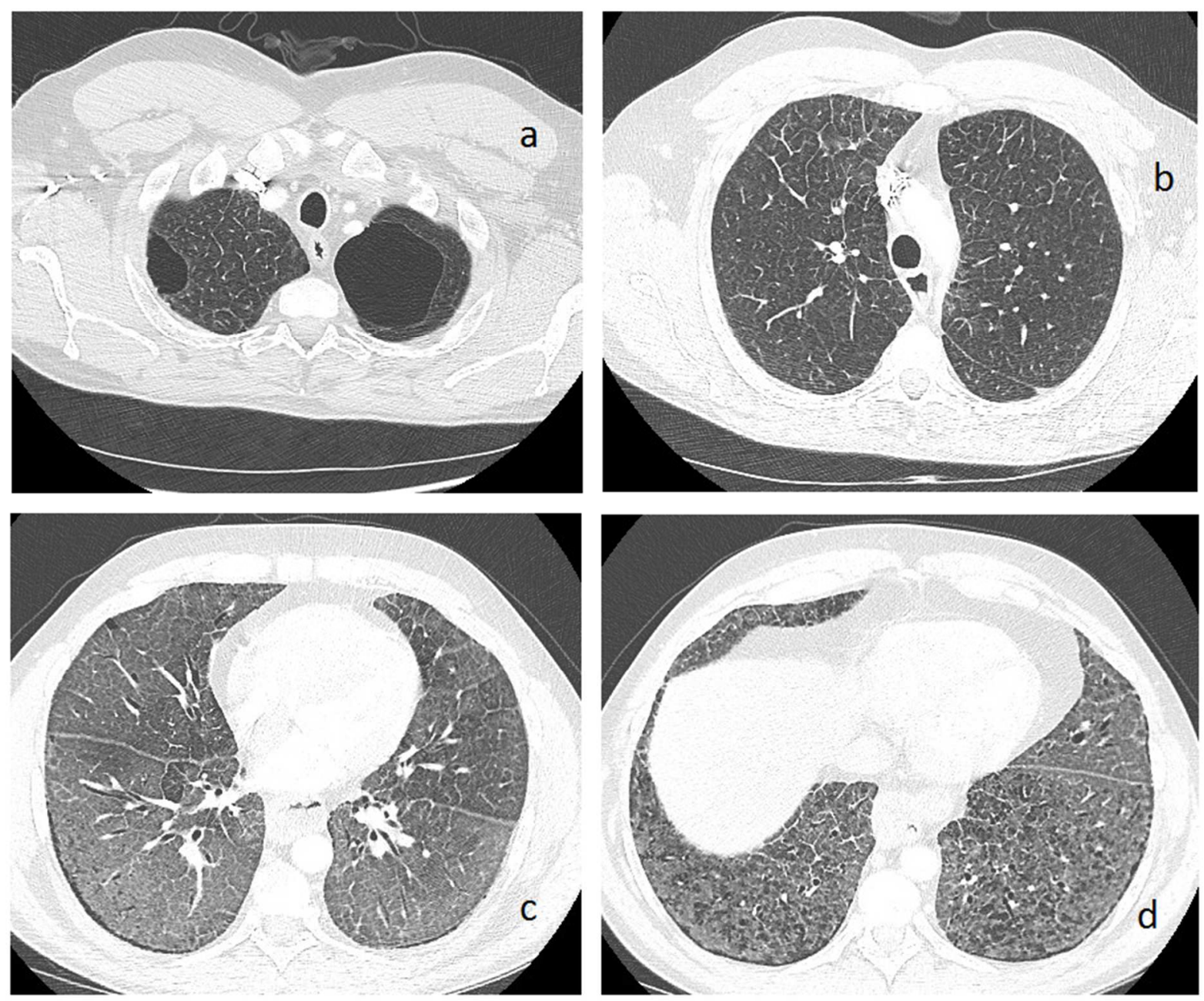

Figure 2: chest computed tomography of case\#2. a: upper lobes with apical emphysema and b: septal thickening. c: middle-lung mixed interlobular and intralobular lines with ground glass opacities. d: lower lobes with ground glass and distal bronchiolectasis.
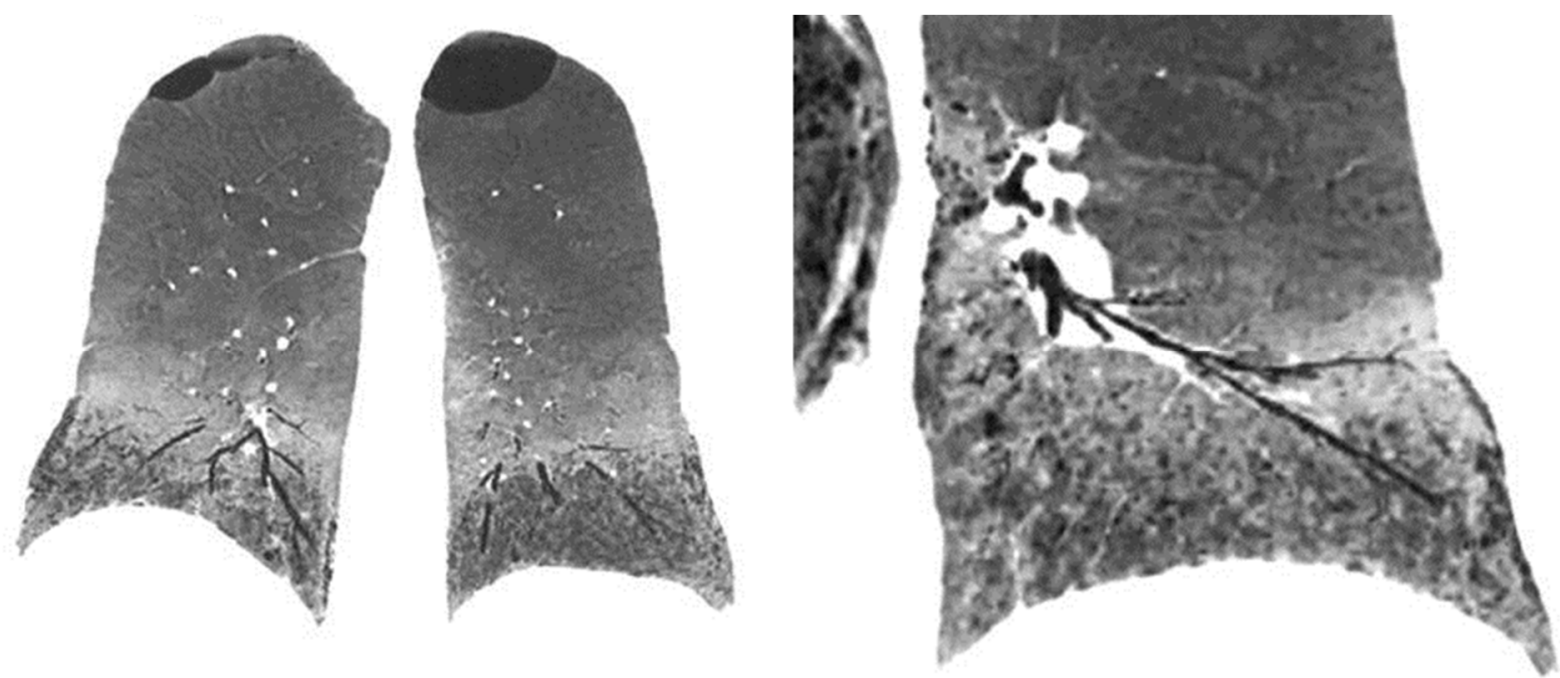

Figure 3: chest computed tomography with minip coronal reconstruction for case\#2. Left: whole lung coronal view illustrating cranio-caudal gradient and variety of lesions. Right: focus 
on lower left lobe highlighting very distal bronchiolectasis.

\begin{tabular}{|c|c|c|}
\hline & Patient \#1 & Patient \#2 \\
\hline Diagnostic age (yrs) & 44 & 3 \\
\hline Red flag symptom & Hepatosplenomegaly & Hepatosplenomegaly \\
\hline $\begin{array}{c}\text { Family cases/SMPD1 } \\
\text { mutations }\end{array}$ & None/homozygous p.R610del & None/in progress \\
\hline $\begin{array}{l}\text { Acid sphingomyelinase } \\
\text { activity }\end{array}$ & Low & Low \\
\hline Main complaint & Dyspnea & Hemoptysis \\
\hline Thrombopenia & Mild & Mild \\
\hline Dyslipidemia & HyperTg, low HDL & HyperTg, low HDL \\
\hline Other biological anomaly & None & Elevated reticulocytes \\
\hline Respiratory impairment & Hypoxemia $\mathrm{PaO}_{2} 55 \mathrm{mmHg}$ & Daily hemoptysis \\
\hline Pulmonary function tests & $\begin{array}{c}\text { Mild restrictive syndrome FVC } 75 \% \text {, } \\
\text { lowered KCO } 45 \%\end{array}$ & $\begin{array}{c}\text { Normal lung volumes, } \\
\text { lowered KCO } 54 \%\end{array}$ \\
\hline Chest imaging & $\begin{array}{l}\text { Mixed interlobular and intralobular } \\
\text { lines, ground-glass opacities }\end{array}$ & $\begin{array}{c}\text { Mixed interlobular and } \\
\text { intralobular lines, ground-glass } \\
\text { opacities }\end{array}$ \\
\hline Extra-thoracic disease & Spleen nodules & None \\
\hline Unusual features & $\begin{array}{c}\text { Subpleural emphysema, } \\
\text { disease exacerbations }\end{array}$ & $\begin{array}{l}\text { Emphysema, } \\
\text { bronchiolectasis, } \\
\text { alveolar hemorrhage }\end{array}$ \\
\hline
\end{tabular}

Table 1: summary of clinical and analyses findings in our two cases. "Red flag symptom" is the symptom that made ASMD diagnosis evoked. "Unusual features" note the original features presented in these cases not reported in previous literature. 


\section{References}

1. Schuchman EH, Desnick RJ. Types A and B Niemann-Pick disease. Mol Genet Metab. févr 2017;120(1-2):27-33.

2. Wasserstein MP, Desnick RJ, Schuchman EH, Hossain S, Wallenstein S, Lamm C, et al. The natural history of type B Niemann-Pick disease: results from a 10-year longitudinal study. Pediatrics. déc 2004;114(6):e672-677.

3. Guillemot N, Troadec C, de Villemeur TB, Clément A, Fauroux B. Lung disease in Niemann-Pick disease. Pediatr Pulmonol. déc 2007;42(12):1207-14.

4. Wasserstein MP, Aron A, Brodie SE, Simonaro C, Desnick RJ, McGovern MM. Acid sphingomyelinase deficiency: prevalence and characterization of an intermediate phenotype of Niemann-Pick disease. J Pediatr. oct 2006;149(4):554-9.

5. McGovern MM, Avetisyan R, Sanson B-J, Lidove O. Disease manifestations and burden of illness in patients with acid sphingomyelinase deficiency (ASMD). Orphanet J Rare Dis. 23 2017;12(1):41.

6. Marten K, Hansell DM. Imaging of macrophage-related lung diseases. Eur Radiol. avr 2005;15(4):727-41.

7. Hervé A, Marchand-Adam S, Fabre A, Debray M-P, Germain D-P, Crestani B, et al. [Niemann-Pick disease type B identified following an episode of bronchopneumonia]. Rev Mal Respir. sept 2008;25(7):861-6.

8. McGovern MM, Wasserstein MP, Giugliani R, Bembi B, Vanier MT, Mengel E, et al. A prospective, cross-sectional survey study of the natural history of Niemann-Pick disease type B. Pediatrics. août 2008;122(2):e341-349.

9. Cox GF, Clarke LA, Giugliani R, McGovern MM. Burden of Illness in Acid Sphingomyelinase Deficiency: A Retrospective Chart Review of 100 Patients. JIMD Rep. 12 juill 2018;

10. Hollak CEM, de Sonnaville ESV, Cassiman D, Linthorst GE, Groener JE, Morava E, et al. Acid sphingomyelinase (Asm) deficiency patients in The Netherlands and Belgium: disease spectrum and natural course in attenuated patients. Mol Genet Metab. nov 2012;107(3):526-33. 11. Minai OA, Sullivan EJ, Stoller JK. Pulmonary involvement in Niemann-Pick disease: case report and literature review. Respir Med. déc 2000;94(12):1241-51.

12. von Ranke FM, Pereira Freitas HM, Mançano AD, Rodrigues RS, Hochhegger B, Escuissato D, et al. Pulmonary Involvement in Niemann-Pick Disease: A State-of-the-Art Review. Lung. 2016;194(4):511-8.

13. Cassiman D, Packman S, Bembi B, Turkia HB, Al-Sayed M, Schiff M, et al. Cause of death in patients with chronic visceral and chronic neurovisceral acid sphingomyelinase deficiency (Niemann-Pick disease type B and B variant): Literature review and report of new cases. Mol Genet Metab. 2016;118(3):206-13.

14. Mendelson DS, Wasserstein MP, Desnick RJ, Glass R, Simpson W, Skloot G, et al. Type B Niemann-Pick disease: findings at chest radiography, thin-section CT, and pulmonary function testing. Radiology. janv 2006;238(1):339-45.

15. Hadda V, Khilnani GC. Lipoid pneumonia: an overview. Expert Rev Respir Med. déc 2010;4(6):799-807.

16. Rossi G, Cavazza A, Spagnolo P, Bellafiore S, Kuhn E, Carassai P, et al. The role of macrophages in interstitial lung diseases: Number 3 in the Series "Pathology for the clinician" 
Edited by Peter Dorfmüller and Alberto Cavazza. Eur Respir Rev. 30 sept 2017;26(145):170009.

17. Freitas HMP, Mançano AD, Rodrigues RS, Hochhegger B, Torres PPTES, Escuissato D, et al. Niemann-Pick disease type B: HRCT assessment of pulmonary involvement. J Bras Pneumol Publicacao Of Soc Bras Pneumol E Tisilogia. déc 2017;43(6):451-5.

18. McGovern MM, Lippa N, Bagiella E, Schuchman EH, Desnick RJ, Wasserstein MP. Morbidity and mortality in type B Niemann-Pick disease. Genet Med Off J Am Coll Med Genet. août 2013;15(8):618-23.

19. Gülhan B, Ozçelik U, Gürakan F, Güçer S, Orhan D, Cinel G, et al. Different features of lung involvement in Niemann-Pick disease and Gaucher disease. Respir Med. sept 2012;106(9):1278-85.

20. McGovern MM, Dionisi-Vici C, Giugliani R, Hwu P, Lidove O, Lukacs Z, et al. Consensus recommendation for a diagnostic guideline for acid sphingomyelinase deficiency. Genet Med Off J Am Coll Med Genet. 2017;19(9):967-74.

21. Nicholson AG, Wells AU, Hooper J, Hansell DM, Kelleher A, Morgan C. Successful treatment of endogenous lipoid pneumonia due to Niemann-Pick Type B disease with whole-lung lavage. Am J Respir Crit Care Med. 1 janv 2002;165(1):128-31.

22. Lau C, Abdelmalak BB, Farver CF, Culver DA. Whole lung lavage for lipoid pneumonia. Thorax. 2016;71(11):1066-7.

23. Wasserstein M, Dionisi-Vici C, Giugliani R, Hwu W-L, Lidove O, Lukacs Z, et al. Recommendations for clinical monitoring of patients with acid sphingomyelinase deficiency (ASMD). Mol Genet Metab. févr 2019;126(2):98-105.

24. McGovern MM, Wasserstein MP, Kirmse B, Duvall WL, Schiano T, Thurberg BL, et al. Novel first-dose adverse drug reactions during a phase I trial of olipudase alfa (recombinant human acid sphingomyelinase) in adults with Niemann-Pick disease type B (acid sphingomyelinase deficiency). Genet Med. janv 2016;18(1):34-40.

25. Lidove O, Mauhin W, London J. Acid sphingomyelinase deficiency (Niemann-Pick disease Type B) as an inflammatory disease. J Heart Lung Transplant. mai 2019;38(5):583-4. 26. Lidove O, Belmatoug N, Froissart R, Lavigne C, Durieu I, Mazodier K, et al. [Acid sphingomyelinase deficiency (Niemann-Pick disease type B) in adulthood: A retrospective multicentric study of 28 adult cases]. Rev Med Interne. mai 2017;38(5):291-9.

27. Mannem H, Kilbourne S, Weder M. Lung transplantation in a patient with Niemann-Pick disease. J Heart Lung Transplant Off Publ Int Soc Heart Transplant. janv 2019;38(1):100-1.

28. Ding F, Mehta AC, Arrossi AV. Successful lung transplantation in a patient with Niemann-Pick disease. J Heart Lung Transplant. mai 2019;38(5):582-3.

29. Wasserstein MP, Caggana M, Bailey SM, Desnick RJ, Edelmann L, Estrella L, et al. The New York pilot newborn screening program for lysosomal storage diseases: Report of the First 65,000 Infants. Genet Med Off J Am Coll Med Genet. 10 août 2018; 